\title{
TIPOS DE ENRAIZADORES EN VARETAS DE (THEOBROMA CACAO), COMUNIDAD CARAO. SIUNA, 2011
}

Freddy Francisco Cordero Rivera ${ }^{[1]}$ Oscar Montalván Castellón ${ }^{[2]}$ Oscar Flores Pérez ${ }^{[3]}$

\section{Resumen}

La presente investigación se realizó en la comunidad Carao-Hormiguero, Siuna, Región Autónoma del Atlántico Norte (RAAN), ubicada en la zona núcleo de la reserva de biosfera BOSAWAS, con el propósito de evaluar la eficacia del proroot y ácido húmico en el enraizamiento en varetas de cacao. El estudio fue experimental, transversal y prospectivo. La metodología basada en el Diseño Completamente al Azar (DCA) con tratamientos de ácido húmico, proroot y testigo en un período de 40 días (20 de septiembre al 30 de octubre). Se utilizaron 180 varetas en 3 tratamientos con 3 réplicas de 60 varetas, 20 por bancal. Con el enraizador ácido húmico se obtuvo un prendimiento de 40\% (24), con Proroot, 68.33\% (41) y testigo, 11.66\% (7). En general se logró un 40\% de prendimiento (72 varetas). La inversión durante la investigación fue de $C \$ 2,548.60$. ( $C \$ 35.39$ por vareta). El tratamiento que dio resultados aceptables fue el proroot, del que se obtiene una ganancia neta de $C \$ 388.80$.

Palabras clave: Proroot; ácido húmico; enraizador; eficacia; beneficio costo.

\section{Summary}

This research was carried out in the Carao Hormiguero community, municipality of Siuna, RAAN, which is located in the center of the Bosawas biosphere reserve, in order to evaluate the effectiveness of ProRoot and the humic acid on the rooting in cacao branches. The research was experimental, transverse and prospective. The methodology was based on a completely randomized design (DCA) with humic acid treatments, ProRoot and control in a period of 40 days (September 20 to October 30 ). 180 branches were used in 3 treatments with 3 replications of 60 branches, 20 by plots. With the rooting humic acid 40\% (24) of capture was obtained, with ProRoot, $68.33 \%$ (41) and control, $11.66 \%$ (7). In general $40 \%$ of capture was achieved (72 branches). The investment during the investigation was of $C \$ 2,548.60$ ( $C \$ 35.39$ per branch). The treatment that gave acceptable results was ProRoot, of which a net profit of $C \$$ 388.80 is obtained.

Keywords: ProRoot; humic acid; rooting; efficacy; benefit-cost.

[1] Ingeniero Agroforestal. Técnico IREMADES. corderoriveraf@yahoo.es

[2] Tutor. Máster en Docencia Universitaria, Técnico IREMADES montesiuna@yahoo.com

[3] Asesor. Oscarflores_7hotmail.com

98 CIENCIA E INTERCULTURALIDAD, Volumen14, Año 7, No. 1, Enero- Junio, 2014 


\section{Introducción}

La multiplicación de varetas de cacao a través de la técnica de enraizamiento, se está desarrollando a escalas considerables en países productores de cacao como Colombia, Brasil, Ecuador, Costa Rica y otros a nivel mundial, los que han tenido éxito al utilizar esta técnica, logrando establecer plantaciones de considerable magnitud, y producciones a corto plazo.

En 1960-1995 en las Regiones de Caldas y Antioquia (Colombia), se inició con el fomento de enraizamiento en varetas como una alternativa de producción a corto plazo y de mejoramiento de la calidad del cacao, donde se comprobó la propagación precoz y bajo impacto ambiental con buenos resultados económicos, en términos genéricos, este método fue realizado y dio buenos éxitos.

En Nicaragua, la técnica de enraizamiento de varetas no es muy común en la reproducción asexual en cacao, especialmente en la región Autónoma Costa Atlántica de Nicaragua. Por esta razón se experimentó este método de producción con el fin de valorar el comportamiento del mismo y evaluar la eficiencia de tipos de enraizadores en varetas de cacao y su costo beneficio.

La manera tradicional (sexual) de replicar el cacao, además de ser un proceso lento ha traído actualmente problemas de degeneración de las semillas, en dicho proceso se da la transferencia de material genético, y con ello también se diseminan las enfermedades que sus progenitores poseen en el momento de la siembra y manejo, ocasionando disminución en la producción del cultivo.

Este estudio aportará beneficios a corto plazo a los productores que realicen enraizamiento en varetas de cacao, tanto en la precocidad de las cosechas como en el mejoramiento genético de sus plantaciones y en la disminución de los costos de establecimiento de cacao por área.

\section{Marco teórico}

\section{Generalidades}

En 1930 se iniciaron las primeras actividades de propagación por estacas en Trinidad. se han utilizado distintos materiales para enraizamiento de estacas, como vermiculita, aserrín de madera descompuesta, fibra de coco, entre otros.

Las estacas deben tomarse en horas de la mañana (6:00 a.m.). Las ramas más indicadas son las abiertas, las de abanicos y los chupones. Se sugiere el empleo de sustancias de crecimiento para favorecer la formación y el desarrollo de las raíces, en especial el ácido beta -indo butírico. (Pilamunga,200o). 


\section{Selección de varetas}

Las estacas utilizadas para el enraizamiento han sido seleccionadas de ramas terminales en abanico, de madera semileñosa, de unos 30-40 cm de largo, o a veces un poco más pequeñas, a las cuales se les cortan sus hojas por la mitad. Estas estacas de ramas se ponen a enraizar bajo camas con techo de vidrio o propagadores. Ibíd.

\section{Eficacia de los enraizadores}

En relación con la función fisiológica de los enraizadores en el proceso de enraizamiento de las varetas en cacao. Una vareta de cacao para enraizar necesita hormonas de crecimiento, estas son las que se encargan de estimular las células y así producir su sistema radicular.

\section{Ácido húmico}

Es un fertilizante natural, producido mediante un proceso de biofermentación, especialmente diseñado para el desarrollo de raíz y potenciar los estados iníciales de desarrollo de los cultivos. La forma de los nutrientes presentes, el alto contenido de ácidos Húmicos y la presencia de 23 aminoácidos son factores que le dan una gran efectividad con respecto a otras fuentes de fósforo y ácidos húmicos (ECOINSUMOS S.A, 2009).

\section{Proroot}

Promueve una mayor producción de raíces y de mejor calidad por lo que se reduce el tiempo de adaptación de las plántulas al momento de ser establecidas en el campo agrícola. Con la aplicación de Proroot se disminuyen las pérdidas de plántulas por trasplante. Se tiene más vigor de las raíces en las etapas iníciales del cultivo lo que derivará en un cultivo bien sostenido y fuerte. Al tener raíces de calidad no solamente se tiene buen inicio del cultivo sino que la planta tiene la capacidad de absorber la humedad necesaria para su rápida adaptación al campo (Ibíd.).

Proroot es un producto especialmente diseñado para inducir y estimular el crecimiento de raíces y el engrosamiento de tallos. Su formulación se basa en una mezcla balanceada de hormonas "enraizadoras", macro nutrimentos y ácidos fúlvicos que actúan para lograr un resultado más rápido y eficaz.

\section{Costo - beneficio de los enraizadores}

Los beneficios y costos socioeconómicos directos de los proyectos de inversión equivalen a los correspondientes valores privados corregidos. Es decir, los costos y beneficios directos. (Fontainer,1,999;P.441). 
Al ratio beneficio / costo también se le conoce como índice de rendimiento, se obtiene dividiendo el valor actual de los flujos de efectivos esperados (entradas de efectivos menos salidas de efectivos) entre el costo total de la inversión inicial (salida de efectivo inicial). (Rodríguez, 2008; p.376).

Costos estimados: Es aquella técnica mediante la cual los costos se calculan sobre ciertas bases empíricas calculando aproximadamente el costo de los elementos que integran antes de producir.

Costos fijos: son aquellos que están en función del tiempo o sea no sufren alteraciones algunas son constante aun cuando se presentan grandes fluctuaciones en el volumen de producción.

Análisis Costo - Beneficio es el proceso de colocar cifras en los diferentes costos $\mathrm{y}$ beneficios de una actividad. Al utilizarlo podemos estimar el impacto financiero acumulado de lo que queremos lograr.

El análisis de Costo - Beneficio (CBA) es el acercamiento de la escala de peso para la toma de decisiones. Todos los elementos positivos (los movimientos de efectivos y otras ventajas intangibles) se ponen en un lado del equilibrio y todos los elementos negativos (los costos y las desventajas) se ponen en el otro. Cualquiera sea el peso, la más pesada gana.

Malespín (2008) realizó un estudio sobre costo en el enraizamiento de ramillas donde para el establecimiento de una manzana de cacao con varetas enraizadas a una distancia de $3 \times 3$ esta se le establece un total de (1111) plantas. Lo cual una ramilla enraizada equivale a (26) córdobas significa que para una manzana solo la compra de ramillas de cacao es de veintiocho mil ochocientos ochenta con seis córdobas netos $(28,886)$ sin meter mano de obra y demás materiales (P. 33-35).

Según un estudio realizado en Colombia por Aldana (2009), refiere que el costo para la producción de mil plantas con el método de enraizamiento de varetas es de 428 dólares equivalente en córdobas $(C \$ 10468.00)$ y dentro de sus principales actividades se cuantificó en selección de árboles, corte de ramillas, corte de hoja y tallo, limpieza de hoja y tallo, aplicación de hormonas, componente de sustrato, aplicación de sustrato, llenado de bolsa, compra de plástico, siembra, postura de plástico y quitado de plástico y reubicación de plántulas (P. 52-56). 


\section{Materiales y métodos}

\section{Localización geográfica del estudio}

El presente estudio, se realizó en la comunidad el Carao-Hormiguero, municipio de Siuna-RAAN, ubicada a 23 Kilómetros al Noreste del área urbana, de Julio- Agosto 2011.

\section{Tipo de estudio}

El estudio es experimental, transversal y prospectivo con un Diseño al azar (DCA).

\section{Universo de estudio}

Árboles de cacao tipo angoleta de 12 años de edad.

\section{Muestra}

180 varetas que fueron distribuidas en 9 bancales, 20 varetas por bancal.

\section{Unidad de análisis}

180 varetas.

\section{Unidad de observación}

Las 180 varetas de cacao.

\section{Variables}

-Eficacia de los enraizadores.

- Costo- beneficio de enraizamiento de varetas en cacao.

\section{Fuentes y obtención de datos:}

\section{Fuentes primarias}

Se obtuvieron mediante la observación de las 180 varetas utilizadas y la recopilación de la información con una guía prediseñada. 


\section{Fuentes secundarias}

Esta se utilizaron manuales, documentos, revistas y diccionarios para definir conceptos con precisión en nuestra investigación.

\section{Técnicas e instrumentos}

\section{Técnica}

Observación directa, sistemática y planificada.

\section{Instrumento}

Formato de registro de campo pre-elaborado para la recopilación de la información de campo.

\section{Resultados y discusión}

\section{Eficacia de los enraizadores}

Los resultados obtenidos en la investigación fueron: tratamiento proroot 41 varetas enraizadas. Tratamiento ácido húmico con 24 varetas enraizadas. Testigo 7 varetas. Estos resultados tienen estrecha relación con lo planteado por Leal (1998), los resultados para enraizamiento de vareta en cacao el mejor es el Proroot. Lo cual refleja que el porcentaje de variabilidad de las unidades experimentales frente al efecto de los tratamientos teniendo como (CV) coeficiente de variación es de $23.57 \%$.

Basados en el análisis estadístico de Fisher con un nivel de significancia del $5 \%$ se encontró que existen diferencias estadísticas significativas entre los tres tratamientos ( $p<0.05$ ), es decir, al menos uno de los tratamientos en estudio, tiene mayor eficiencia en el enraizamiento de varetas de cacao. Dado que la prueba de Fisher ( $\mathrm{p}<0.05)$ demostró que al menos un efecto de un tratamiento es distinto de los demás, se recurrió a la prueba de Duncan para determinar cuál de los tratamientos es más eficiente en el enraizamiento de varetas de cacao, de ahí que el Proroot hace la diferencia entre los tratamientos en estudio.

Un estudio realizado por Leal, Tal. (1998), también encontró que el Proroot es mejor en el enraizamiento de vareta en cacao, por lo tanto las conclusiones estadísticas deben ser interpretadas con cautela, dado que el coeficiente de variación calculado supera la variabilidad tolerada para un Diseño Completamente al Azar (<5\%). Ello significa que el material experimental y el manejo del área experimental, proporcionaron la suficiente homogeneidad de las unidades experimentales. 
Cuadro No. 1. Eficacia de los enraizadores en varetas de cacao

\begin{tabular}{|l|l|l|l|}
\hline Varetas por bancal & $\begin{array}{c}\text { Varetas enraizadas } \\
\text { con Proroot }\end{array}$ & $\begin{array}{l}\text { Varetas enraizadas } \\
\text { con ácido húmico }\end{array}$ & $\begin{array}{c}\text { Varetas enraizadas } \\
\text { con Testigo }\end{array}$ \\
\hline 20 & 13 & 8 & 1 \\
\hline 20 & 12 & 10 & 2 \\
\hline 20 & 16 & 6 & 4 \\
\hline Total & 41 & 24 & 7 \\
\hline \% enraizamiento & $\mathbf{6 8 . 3 3}$ & $\mathbf{4 0}$ & $\mathbf{1 1 . 6 6}$ \\
\hline
\end{tabular}

Con respecto los porcentajes de enraizamientos se pudieron notar una mayor eficiencia en varetas jóvenes de 30 y 60 días, son las mejores para el enraizamiento de ramillas (80\% de enraizamiento), estos resultadosse relacionan con lo referido por Wargadipura, (1986) y Catálogo ORTON, IICA, CATIE octubre, 2007),Evans et, al 19511976 quienes las estacas utilizadas para un mayor enraizamiento han sido seleccionadas de ramas terminales obteniendo un $60 \%$ de confiabilidad en relación con las demás varetas. En el estudio se utilizaron ramillas abiertas y de abanico y estimulantes de crecimiento de raíces, con resultados que se relacionan con lo planteado por Alberto y Batista quien explica que las ramas más indicadas para el enraizamiento son las abiertas, las de abanicos los chupones. Obteniendo un prendimiento de $20 \%$ en ramillas abiertas, $68 \%$ en ramillas abanico y un $46 \%$ en chupones, Se sugiere el empleo de sustancias de crecimiento para favorecer la formación y el desarrollo de las raíces

\section{Costos-beneficios del enraizamiento en varetas de cacao}

De acuerdo con estimaciones de mercado el precio unitario por varetas es de $C \$ 30.00$ córdobas.En el estudio se obtuvieron ganancias con el enraizador proroot de $C \$ 388.8$. Aldana (2009) refiere que el costo para la producción de mil plantas con el método de enraizamiento de varetas es de $(\$ 1087.00)$ y dentro de sus principales actividades se cuantificó en selección de árboles, corte de ramillas, corte de hoja y tallo, limpieza de hoja, mano de obra y compra de hormonas y aplicación. Estos datos se relacionan con nuestra investigación ya que los costos de producción de una vareta son de $C \$$ 20.5 enraizada con proroot. cuadro 2.

De acuerdo con lo anteriormente descrito el análisis costo beneficio del uso de proroot como enraizador resulta rentable $(\mathrm{B} / \mathrm{C}>1)$ por tanto genera aporte económico adicional. Mientras que los demás tratamientos no hay una recuperación de lo invertido, lo que significa que el estudio con las 60 varetas por cada uno de ellos no es rentable. 
Cuadro No. 2. Costos-beneficios del enraizamiento en varetas de cacao

\begin{tabular}{|l|l|l|l|l|}
\hline \multicolumn{1}{|c|}{ Descripción } & \multicolumn{1}{|c|}{ Proroot } & ácido húmico & \multicolumn{1}{c|}{ Testigo } & \multicolumn{1}{c|}{ Total } \\
\hline Varetas enraizadas & 41 & 24 & 7 & 72 \\
\hline Costo & $\mathrm{C} \$ 841.2$ & $\mathrm{C} \$ 966.2$ & $\mathrm{C} \$ 741.2$ & $\mathrm{C} \$ 2548.6$ \\
\hline Beneficio & $\mathrm{C} \$ 1230.00$ & $\mathrm{C} \$ 720.00$ & $\mathrm{C} \$ 210.00$ & $\mathrm{C} \$ 2160.00$ \\
\hline Costo de varetas enraizadas & $\mathrm{C} \$ 20.5$ & $\mathrm{C} \$ 40.26$ & $\mathrm{C} \$ 105.9$ & \\
\hline Ganancia neta & $\mathbf{3 8 8 . 8}$ & $\mathbf{- 2 4 6 . 2}$ & $\mathbf{- 5 3 1 . 2}$ & \\
\hline
\end{tabular}

\section{Conclusiones}

El proroot ( $\mathrm{p}<0.05)$ es más eficaz en el enraizamiento de varetas de cacao de 30 días de establecidas. En costo- beneficio el tratamiento con mejores resultados obtenido en este estudio es el proroot, lo cual indica que el estudio sobre pasa el nivel de productividad reflejado en el punto de equilibrio. La producción promedio de varetas enraizadas en todo el estudio fue de un $40 \%$ de prendimiento en los nueve bancales sometidos a estudio.

\section{Recomendaciones}

A los investigadores Probar la eficacia del proroot en el enraizamiento con los tipos de cacao más comunes en la región como cundeamor, amelonado, lagarto, calabacín. Probar la capacidad de enraizamiento de ácido húmico y Proroot en varetas con diferentes edades

\section{Lista de referencia}

Pilamunga, S. (2000). Selección del tiempo para la recolección de ramillas de cacao para enraizar, Barranquilla, Colombia.

ECOINSUMOS.S.A. (05 de Junio de 2009). Fertilizantes ecológicos productos. Recuperadoel11deOctubrede2011, de Enraizador acido Húmico: http:// www.ecoinsumos.com/productos-enraizador-humico.htm

Fontainer, E. (1999). Evaluación social de proyecto. Edit. Alfaomega. $12^{\mathrm{a}} \mathrm{ed}$.

Rodríguez, V. (2008). Formulación y evaluación de proyecto. Edit. Alfa Omega.

Malespín, M.(2008). Costo para establecimiento de una manzana con varetas enraizadas, Waslala, Nicaragua.

Aldana, E. (2009). Costo-beneficio en enraizamiento de varetas, Colombia. Braudeau, J. (1979). El Cacao, 185-234. 